\title{
Magnolol Affects Cellular Proliferation, Polyamine Biosynthesis and Catabolism-Linked Protein Expression and Associated Cellular Signaling Pathways in Human Prostate Cancer Cells in vitro
}

\author{
Brendan T. McKeown and Robert A. R. Hurta* \\ Department of Biology, University of Prince Edward Island, 550 University Ave., \\ Charlottetown, PE, Canada, C1A4P3
}

*Corresponding Author: Robert A. R. Hurta, PhD, Department of Biology, University of Prince Edward Island, 550 University Avenue, Charlottetown, PE, Canada, C1A 4P3

Submission date: August 11, 2014; Acceptance date: January 7, 2015; Publication date: January 8, 2015

\begin{abstract}
:
Background: Prostate cancer is the most commonly diagnosed form of cancer in men in Canada and the United States. Both genetic and environmental factors contribute to the development and progression of many cancers, including prostate cancer.

Context and purpose of this study: This study investigated the effects of magnolol, a compound found in the roots and bark of the magnolia tree Magnolia officinalis, on cellular proliferation and proliferation-linked activities of PC3 human prostate cancer cells in vitro.

Results: PC3 cells exposed to magnolol at a concentration of $80 \mu \mathrm{M}$ for 6 hours exhibited decreased protein expression of ornithine decarboxylase, a key regulator in polyamine biosynthesis, as well as affecting the expression of other proteins involved in polyamine biosynthesis and catabolism. Furthermore, protein expression of the R2 subunit of ribonucleotide reductase, a key regulatory protein associated with DNA synthesis, was significantly decreased. Finally, the MAPK (mitogen-activated protein kinase), PI3K (phosphatidylinositol 3-kinase), NFKB (nuclear factor of kappa-light-chain-enhancer of activated B cells) and AP-1 (activator protein 1) cellular signaling pathways were assayed to determine which, if any, of these pathways magnolol exposure would alter. Protein expressions of p-JNK-1 and c-jun were significantly increased while p-p38, JNK-1/2, PI3Kp85, p-PI3Kp85, p-Akt, NFкBp65, p-IкB $\alpha$ and $\mathrm{I} \kappa \mathrm{B} \alpha$ protein expressions were significantly decreased.
\end{abstract}

Conclusions: These alterations further support the anti-proliferative effects of magnolol on PC3 human prostate cancer cells in vitro and suggest that magnolol may have potential as a novel anti-prostate cancer agent.

Key Words: prostate cancer cells, magnolol, polyamines, MAPK, PI3K, NFкB 


\section{INTRODUCTION:}

Prostate cancer is the most commonly diagnosed form of cancer in men in Canada and the United States, representing roughly $10 \%$ of cancer related deaths in men in both countries [1,2]. As a result, there is an increased interest in the research of natural products, many of which have traditionally been used in herbal medicines, and their bioactive effects and potential chemoprotective and chemopreventative capacities [3-5].

Magnolol, a lignan found in the roots and bark of the magnolia tree, Magnolia officinalis, has previously been shown to affect important cellular processes, such as the cell cycle and apoptosis in a variety of human cancer cell types both in vitro and in vivo [6-14]. Those studies performed in vivo suggest that magnolol remains active when administered at a sufficient oral dosage. Animal models have been used to determine the bioavailability of magnolol using two delivery methods: oral administration and intravenous injection. It was found that an injected dose of 2-10 mg/kg could achieve a maximum blood plasma concentration of $10 \mu \mathrm{g} / \mathrm{ml}$ or $40-\mu \mathrm{M}$ magnolol, while oral administration of $20 \mathrm{mg} / \mathrm{kg}$ could achieve a maximum blood plasma concentration of $0.1 \mu \mathrm{g} / \mathrm{ml}$ or $0.4-\mu \mathrm{M}$ magnolol [15-17]. Magnolol has also been shown to be non-toxic and to have no drug related side effects at any dose including the highest tested doses in several studies [15, 18, 19]. These previously observed effects warrant further investigation into the mechanisms by which the cell responds to the anticancer effects of magnolol.

Polyamines (putrescine, spermidine and spermine) are essential to various cellular processes, particularly cellular growth, and the levels of polyamines are carefully controlled within a narrow range [20]. These compounds can bind to RNA, DNA, and proteins to change their functions or to increase their expression; one of the most common ways in which polyamines affect cells is in their binding to mRNA and stimulating protein synthesis by causing structural changes [21]. The biosynthesis of polyamines is controlled by ornithine decarboxylase (ODC), a rate limiting factor in the conversion of ornithine to putrescine. S-adenosylmethionine decarboxylase (SAMDC) is then responsible for further biosynthesis from putrescine to spermidine and from spermidine to spermine. Two further proteins, polyamine oxidase (PAO) and spermidine/spermine N1-acetyltransferase (SSAT), (both of which are capable of converting spermine to spermidine and spermidine to putrescine), control polyamine catabolism. Elevated polyamine levels have been associated with prostate cancer and altered expression of the rate limiting enzymes involved in both polyamine biosynthesis and catabolism. These are often observed during the development of cancer [22]. In particular, ODC expression is generally increased in human cancers and therapies targeting these polyamine biosynthetic/catabolic enzymes. This has been used in both chemotherapy and in chemoprevention [22].

In the present study, the in vitro effects of magnolol on the expression of proteins involved in the biosynthesis and catabolism of polyamines in PC3 human prostate cancer cells were examined. This is novel research, as the effects of magnolol on this polyamine pathway have not yet been investigated. Furthermore this investigation seeks to determine the degree to which polyamine related proteins are modulated in response to magnolol exposure and to characterize the cellular signal transduction mechanisms involved in the effect of magnolol on cellular proliferation. 


\section{METHODS AND MATERIALS:}

Materials: All antibodies used in this study were purchased from Santa Cruz Biotechnology, Inc. (Santa Cruz, CA). These include: goat polyclonal anti-ODC, anti-AZ, anti-R1, anti-R2, antip-JNK, anti-JNK-1, anti-p-PI3Kp85, anti-Akt, anti-Histone $\mathrm{H} 2 \mathrm{~B}$ and anti-p-IкB $\alpha$, mouse polyclonal anti-actin, anti-PAO, anti-SAMDC, anti-PI3Kp85 and anti-I $\mathrm{B} \alpha$, and rabbit polyclonal anti-SSAT, anti-AZI, anti-p-ERK, anti-ERK-1, anti-ERK-2, anti-p-p38, anti-p38, anti-JNK-2, anti-PI3Kp110, anti-p-Akt, anti-NFkBp65, anti-c-jun and anti-c-fos. All other chemicals and materials were purchased from Sigma-Aldrich Canada (Oakville, ON) unless otherwise indicated.

Cell Culture and Treatment with Magnolol: Human PC3 prostate adenocarcinoma cells (ATCC, Manasses, VA) were cultured on $100 \mathrm{~mm}$ plastic tissue-culture dishes (Falcon, Mississauga, ON) in RPMI (Gibco, Burlington, ON). These were supplemented with 10\% fetal bovine serum (FBS) and 1\% antibiotic-antimycotic (Invitrogen Canada, Burlington, ON), and were incubated at $37^{\circ} \mathrm{C}$ in $5 \% \mathrm{CO}_{2}$. At $70 \%$ confluence. The cells were exposed to $80 \mu \mathrm{M}$ concentrations of magnolol, dissolved in dimethyl sulfoxide (DMSO), for 6 hours (concentration of DMSO in both magnolol-treated and control cells was $0.2 \%(\mathrm{v} / \mathrm{v}))$. Control cells received only DMSO. After treatment, cells were removed by trypsin diluted in phosphate buffered saline (PBS) and re-suspended in RPMI with 10\% FBS and centrifuged for $5 \mathrm{~min}$ at $500 \mathrm{X}$ g. The media was then removed by aspiration. The remaining cell pellet was re-suspended and washed in cold PBS. Cells were again centrifuged for $5 \mathrm{~min}$ at 500X g. After centrifugation, the PBS was removed by aspiration and the pellet was then stored at $-80^{\circ} \mathrm{C}$ until further analysis.

Cellular Growth (Proliferation) Curve: PC3 human prostate adenocarcinoma cells were subcultured at 25,000 cells/plate on $100 \mathrm{~mm}$ plastic tissue culture dishes in RPMI supplemented with $10 \%$ FBS and $1 \%$ antibiotic-antimycotic. Following 24 hours of incubation, cells were counted using a haemocytometer to determine cell number at the 0 hour time point. Cells were then treated with either $80 \mu \mathrm{M}$ magnolol or DMSO (vehicle). Cells were again counted at 6, 24, 48 and 72-hour time points. Triplicate plates were counted for each treatment (control or $80 \mu \mathrm{M}$ magnolol) at each time point.

Nuclear and Cytosolic Protein Fraction Isolation: Nuclear and cytosolic protein isolation was performed using a nuclear extraction kit (Cayman Chemical, Ann Arbor, MI) as per manufacturer's instruction. Briefly, cells were treated and pelleted as above, then cell pellets were suspended in $250 \mu \mathrm{L}$ of hypotonic buffer (10\% 10x hypotonic buffer, $2 \%$ phosphatase inhibitors, $1 \%$ protease inhibitors, $87 \%$ distilled water) and incubated on ice for 15 min. After 15 min, $50 \mu \mathrm{L}$ of $10 \%$ Nonident P-40 assay reagent was added to each sample and mixed by pipetting. Then samples were centrifuged at $14000 \mathrm{X} \mathrm{g}$ for $30 \mathrm{sec}$ at $4^{0} \mathrm{C}$. The supernatant was then removed and stored at $-80^{\circ} \mathrm{C}$, as this represents the cytosolic fraction. The remaining cell pellet was then resuspended in $50 \mu \mathrm{L}$ of extraction buffer $(50 \% 2 \mathrm{x}$ nuclear extraction buffer, $2 \%$ phosphatase inhibitors, $1 \%$ protease inhibitors, $37 \%$ distilled water) and vortexed for $15 \mathrm{sec}$, then shaken for $15 \mathrm{~min}$ on ice. Samples were again vortexed, for $30 \mathrm{sec}$, and shaken a second time for $15 \mathrm{~min}$ on ice. Finally, samples were centrifuged at $14000 \mathrm{X} \mathrm{g}$ for $10 \mathrm{~min}$ at $4^{0} \mathrm{C}$ and the 
supernatant, representing the nuclear fraction, was removed and stored at $-80^{\circ} \mathrm{C}$ until further immunoblot analysis.

Immunoblot Analysis: Cell pellets (for those samples not undergoing nuclear isolation) were resuspended in $100 \mu \mathrm{l}$ of $10 \mathrm{mM}$ Tris-HCl buffer $(\mathrm{pH}$ 7.4) containing $0.5 \mathrm{mM}$ phenylmethanesulfonylfluoride (PMSF), and briefly sonicated. Cell lysates were then centrifuged at 9,300X $\mathrm{g}$ for $20 \mathrm{~min}$ at $4^{\circ} \mathrm{C}$. The supernatant was removed from the pellet as this represents a whole cell lysate sample. Nuclear, cytosolic and whole lysate samples were evaluated for protein content. Equal amounts of protein from these extracts were mixed in a 3:1 ratio with standard Laemmli buffer consisting of $50 \mathrm{mM}$ Tris- $\mathrm{HCl}(\mathrm{pH} 6.8), 10 \% \mathrm{SDS}, 0.1 \%$ bromophenol blue, $10 \%$ glycerol and $100 \mathrm{mM} \beta$-mercaptoethanol and boiled for $3 \mathrm{~min}$. Electrophoresis through $10 \%$ SDS-PAGE gels was used to resolve proteins which were then transferred onto nitrocellulose membranes (Bio-Rad, Mississauga, ON) by electro-blotting. Membranes were then incubated in the presence of a $1 \%$ BSA (w/v) TBS-Tween $(0.05 \% \mathrm{v} / \mathrm{v})$ solution overnight at $4^{0} \mathrm{C}$. Primary antibodies diluted to $1: 200(\mathrm{v} / \mathrm{v})$ were then applied to membranes and incubated for 1 hour at room temperature. After incubation, the membranes were washed three times with TBS-Tween $(0.05 \% \mathrm{v} / \mathrm{v})$ for a total of $30 \mathrm{~min}$ then incubated with alkaline phosphatase (AP)-conjugated secondary antibodies (1:1000) for 1.25 hours at room temperature. After incubation the membranes were again washed three times with TBS-Tween $(0.05 \%)$ for a total of $30 \mathrm{~min}$ and rinsed quickly with distilled water, then exposed to SigmaFast BCIP-NBT tablets (Sigma-Aldrich, Oakville, ON) dissolved in distilled water. This was done to visualize protein expression levels. Western blots were then photographed using Infinity Capture Software (Lumenera Corp., Ottawa, ON), and densitometry was performed with ImageJ Software (National Institute of Health, Bethesda, MD).

Statistical Analysis: Statistical analysis was conducted using GraphPad Prism 4.03 for Windows (GraphPad Software, Inc. San Diego, CA). Cell counts in the cellular growth (proliferation) curve were compared to the corresponding cell count for control cells at the appropriate time point using a simple t-test. Results were considered statistically significant at $P \leq 0.1$. Results of immunoblot analysis were compared using a simple t-test and results were considered statistically significant at $P \leq 0.1$.

\section{RESULTS:}

Magnolol Affects Cellular Proliferation: It has previously been shown that magnolol is cytotoxic to PC3 human prostate cancer cells in vitro $[11,23]$. To confirm that this cytotoxicity is accompanied by reduced cellular proliferation, a cellular growth curve experiment was conducted (Figure 1). Time course experiments show that cellular proliferation is retarded in PC3 cells exposed to $80 \mu \mathrm{M}$ magnolol. A magnolol concentration of $80 \mu \mathrm{M}$ has been previously shown to be an effective concentration $[11,23]$. At 6 hours exposure to magnolol there was no significant difference in cellular proliferation between magnolol exposed and control cells; however, by 24 hours a statistically significant difference in cell numbers was observed whereby control cell count increased to $231 \%$ of 0 hour cell count and the magnolol exposed cell count only increased to $151 \%$ of 0 hour cell count. Cellular proliferation in magnolol exposed cells was 
essentially stopped at 48 hours where cell counts were $154 \%$ of 0 hour counts- only a $2 \%$ increase from 24 hour counts. By 72 hours magnolol exposed cell counts were lower than the 0 hour count while control cells counts were at $714 \%$ of 0 hour counts.

\section{Growth Curve}

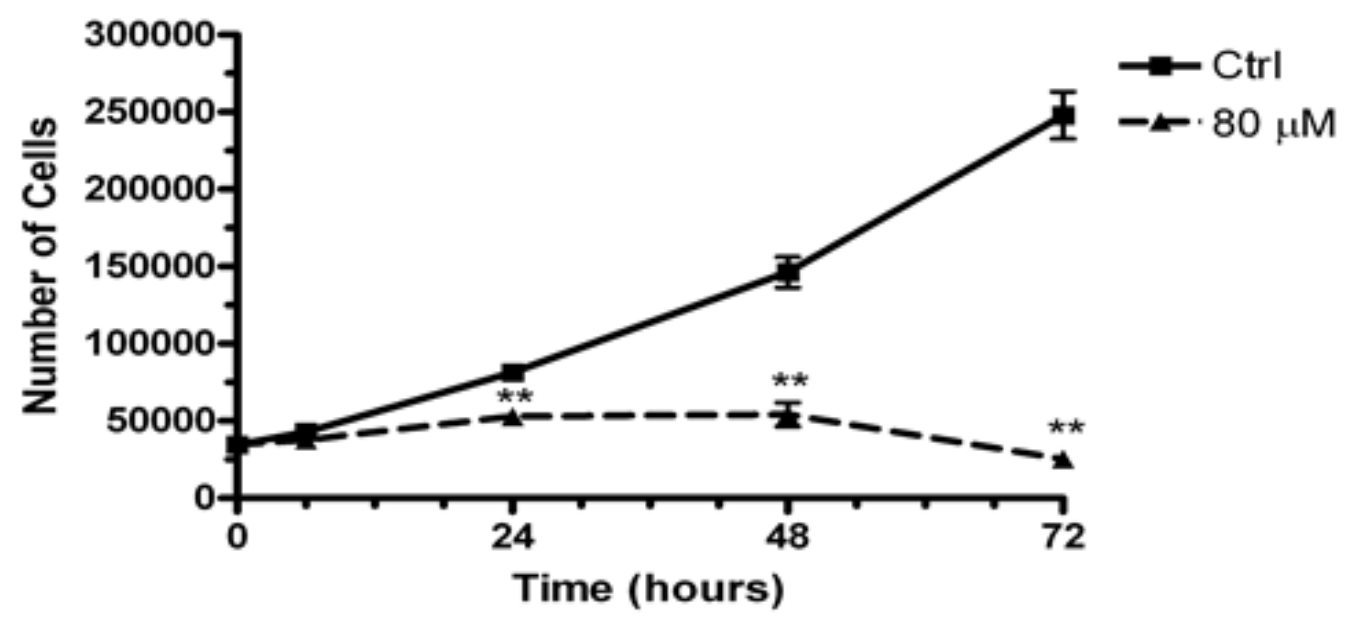

Figure 1. Magnolol affects cellular proliferation of PC3 human prostate cancer cells in vitro. Cells were treated with either DMSO (Ctrl) or $80 \mu \mathrm{M}$ magnolol for 6, 24, 48 or 72 hours. The number of cells present under these conditions was determined in three separate experiments for each time point. Statistical significance between control and magnolol treated cells was determined at each time point. (*) indicates statistical significance at $P \leq 0.1$ and $(* *)$ indicates statistical significance at $P \leq 0.05$.

Magnolol Affects Proliferation Linked Activities: Having established that magnolol exposure results in decreased cellular proliferation, expression of proteins involved in two important proliferation-linked activities were examined. In PC3 cells exposed to $80 \mu \mathrm{M}$ magnolol for 6 hours the protein expression of ornithine decarboxylase (ODC), antizyme inhibitor (AZI), spermidine/spermine N1-acetyltransferase (SSAT) and polyamine oxidase (PAO) were significantly decreased by exposure to magnolol, while protein expression of $\mathrm{S}$ adenosylmethionine decarboxylase (SAMDC) was significantly increased (Figure 2).

Antizyme (AZ) protein expression remained unchanged (Figure 2). While ODC protein expression decreased to $68 \%$ of control and AZI protein expression decreased to $73 \%$ of control, protein expression of SAMDC increased to $154 \%$. Of those proteins directly involved in polyamine catabolism, SSAT protein expression decreased to $66 \%$ of control and PAO protein expression decreased to $43 \%$ of control.

To further determine the effect of magnolol on cellular proliferation in PC3 cells, the two subunits of ribonucleotide reductase ( $\mathrm{R} 1$ and $\mathrm{R} 2$ ) (which is a key rate limiting and regulatory activity of DNA synthesis) were assayed (Figure 3). As ribonucleotide reductase is necessary for the synthesis of deoxyribonucleoside triphosphates, which are the building material for DNA, changes in protein expression of the either R1 or R2 or both subunits would represent an important pathway by which cellular proliferation may be affected [24, 25]. Since ribonucleotide reductase is tightly linked to DNA synthesis, changes to either subunit or both would suggest changes to DNA synthesis. While R1 protein expression was not significantly affected by magnolol exposure the protein expression of R2 decreased to $62 \%$ of control. 
A

ODC

SSAT

$\mathrm{Az}$

Actin
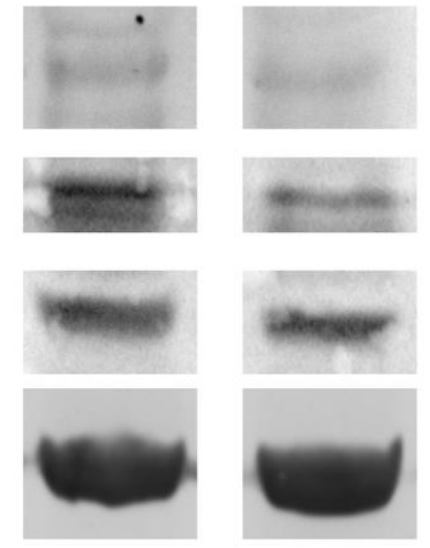

$B$

\section{Ctrl}

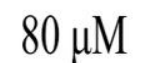

Azl

Actin

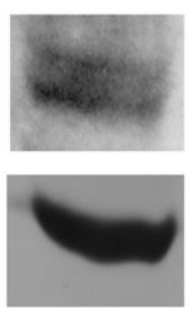

Ctrl

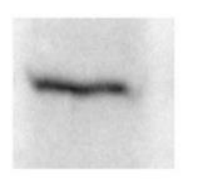

SAMDC

Actin
D ODC
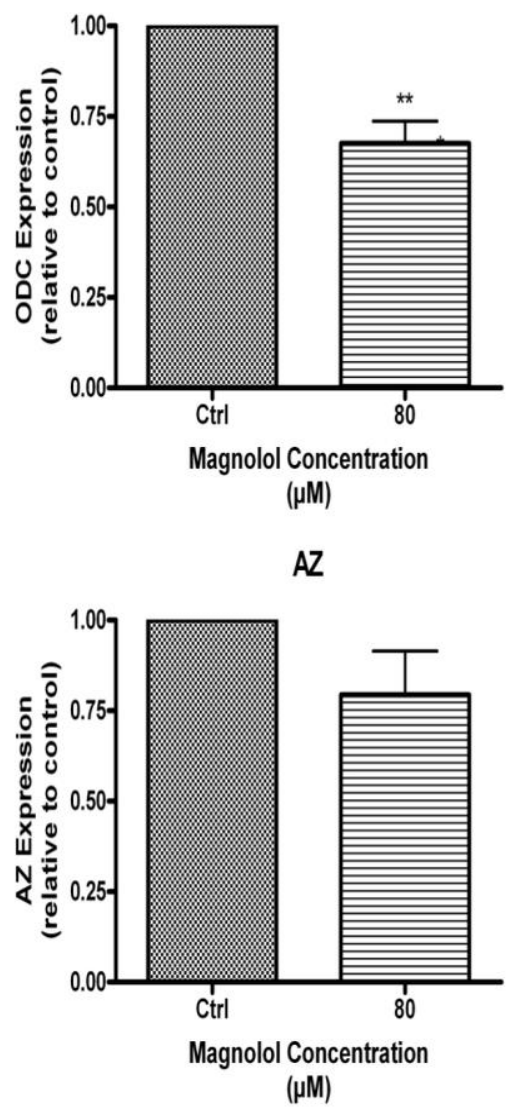

PAO

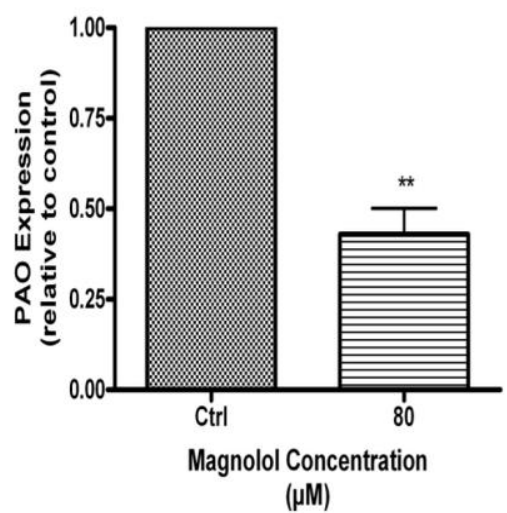

SSAT

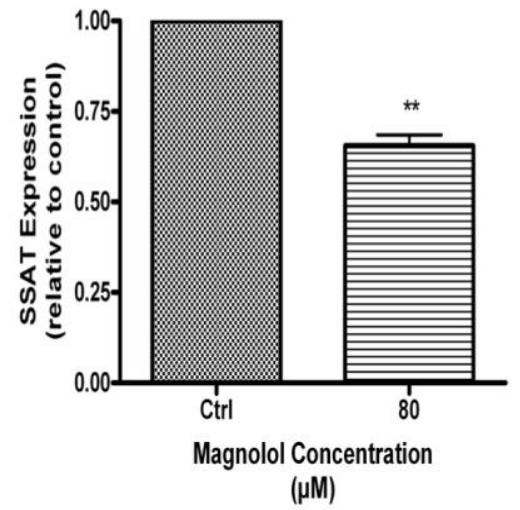

AZI

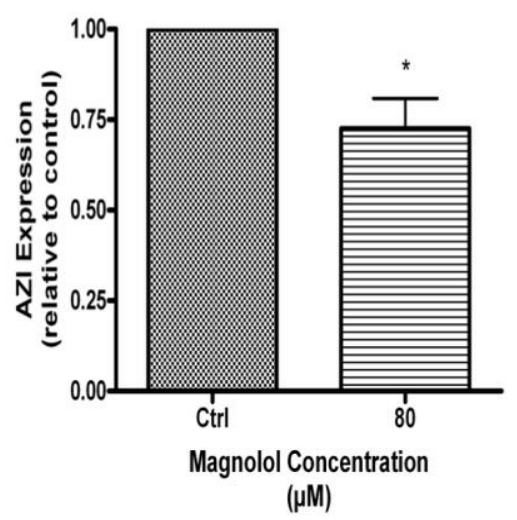

SAMDC

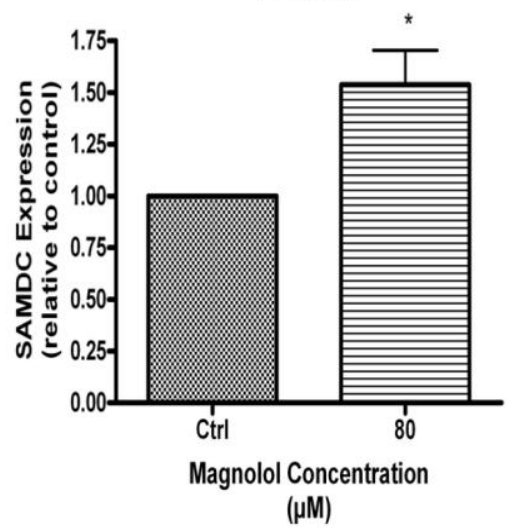

Figure 2(A-D). Magnolol affects expression of polyamine biosynthesis/catabolism-linked proteins in PC3 cells in vitro. Cells were treated with either DMSO (Ctrl) or $80 \mu \mathrm{M}$ magnolol for 6 hours. Actin was used as a loading control (the actin used in Figure 1A is the same as those used in Figures 2B, 3B and 4B; the actin used in Figure 1B is the same as those used in Figures 2A and 4C, as these samples were run concurrently). Each blot shown is representative of results obtained from at least three replications from three separate experiments.

A, B and C: Representative Western blots of proteins involved in polyamine biosynthesis and catabolism.

D: Graphical representation of protein expression. $(*)$ indicates statistical significance at $P \leq 0.1$ and $(* *)$ indicates statistical significance at $P \leq 0.05$. 


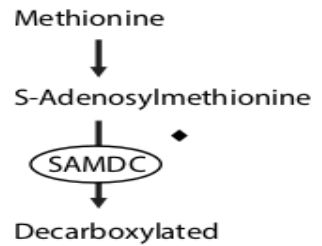

S-Adenosylmethionine

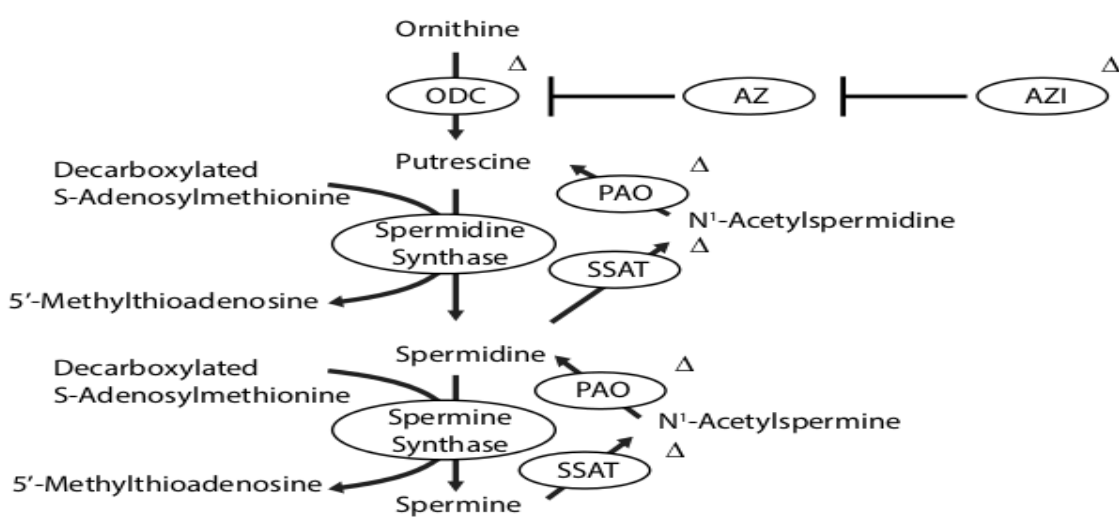

$\longrightarrow$ Activation
$\longrightarrow$ Inhibition

Figure 2E: A diagram which illustrates the proposed sites of action (effects) of magnolol on activities associated with polyamine biosynthesis and catabolism in PC3 human prostate cancer cells. Open triangles indicate that magnolol decreases the expression levels of these proteins, whereas the closed diamond indicates that magnolol increases the expression evels of this protein. AZ protein expression levels were unaffected by magnolol.
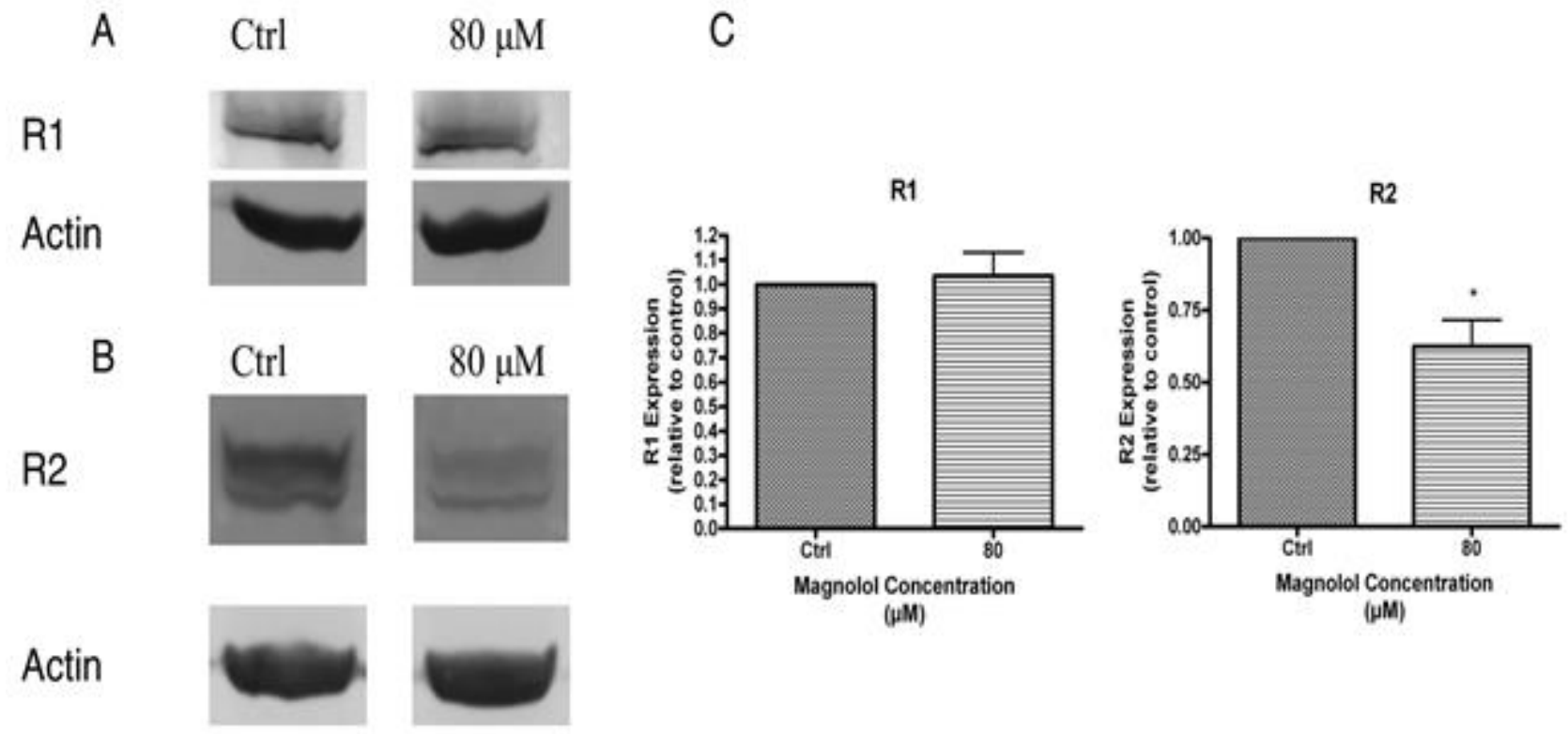

Figure 3. Magnolol affects expression of R2 subunit of ribonucleotide reductase in PC 3 cells in vitro. Cells were treated with either DMSO (Ctrl) or $80 \mu \mathrm{M}$ magnolol for 6 hours. Actin was used as a loading control (the actin used in Figure 2A is the same as those used in Figures 1B and 4C; the actin used in 2B is the same as those used in Figures 1A, 3B and 4B, as these samples were run concurrently). Each blot shown is representative of results obtained from at least three replications from three separate experiments.

A and B: Representative Western blots of the R1 and R2 proteins, respectively.

C: Graphical representation of protein expression. $\left({ }^{*}\right)$ indicates statistical significance at $\mathrm{P} \leq 0.1$ and $(* *)$ indicates statistical significance at $\mathrm{P} \leq 0.05$. 
A

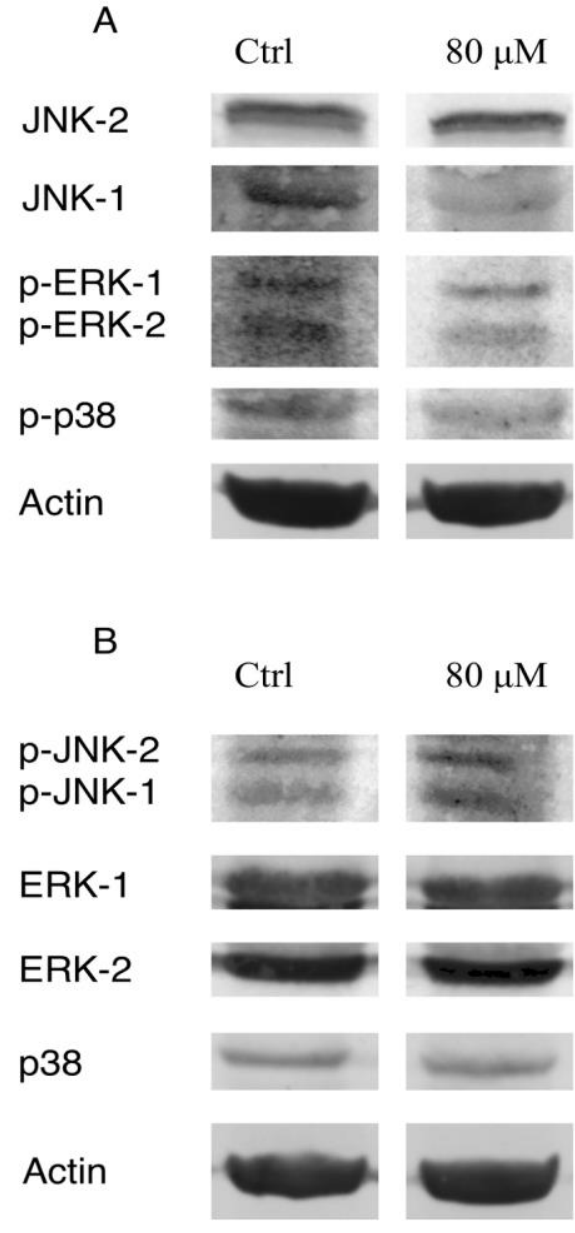

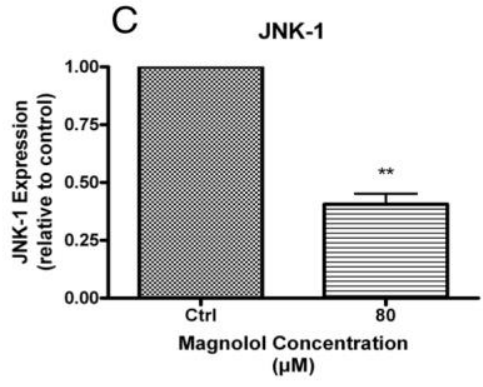

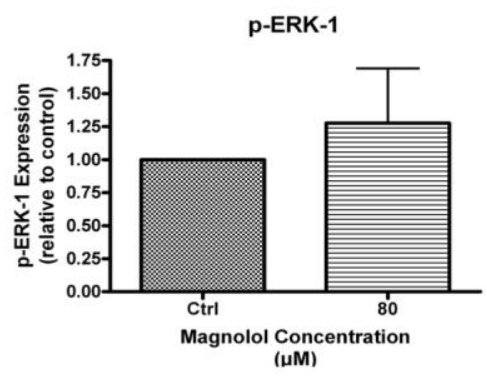

p-p38
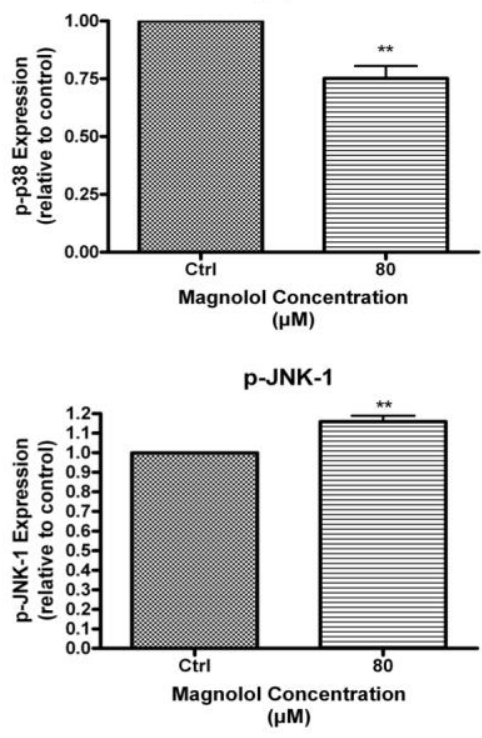

ERK-2

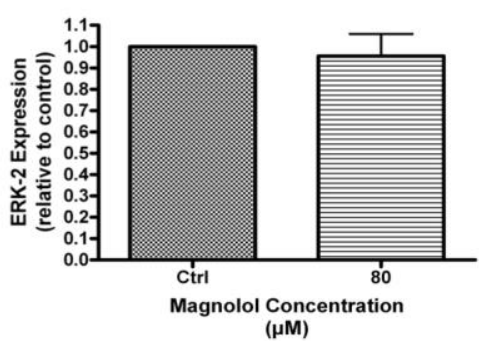

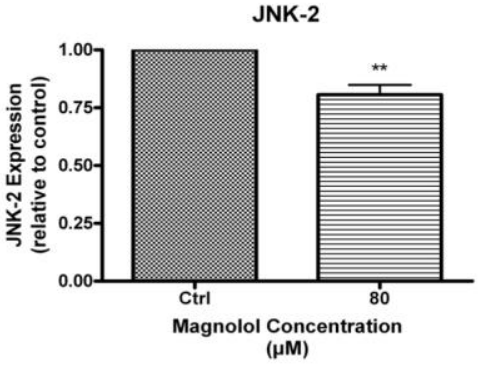
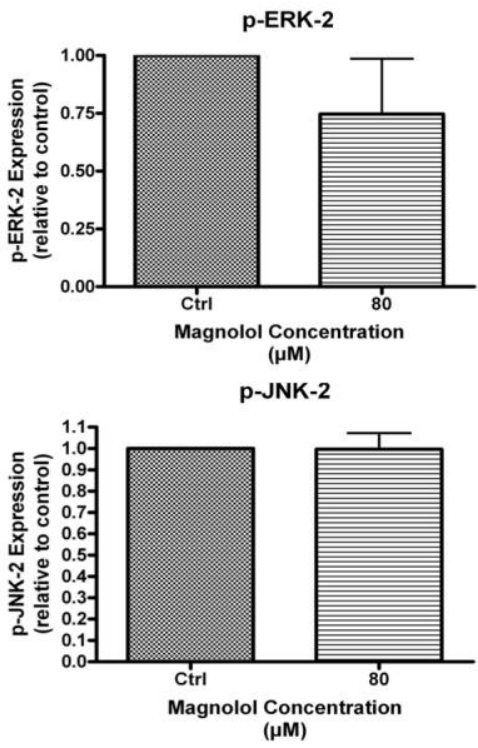

ERK-1

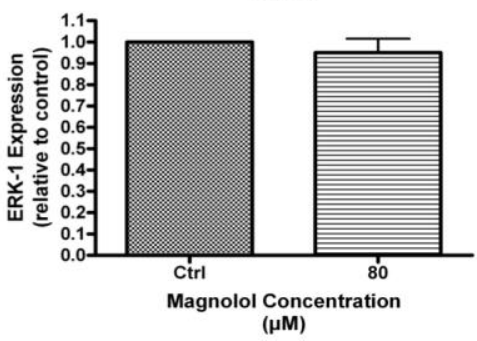

p38

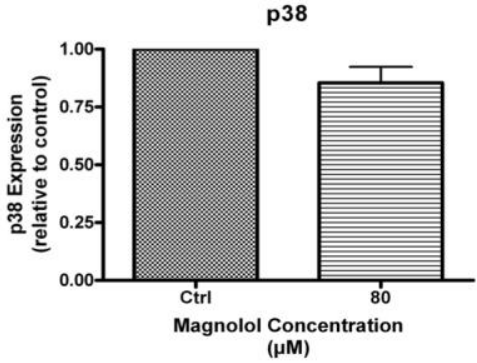

Figure 4. Magnolol affects expression of MAPK pathway proteins in PC3 cells in vitro. Cells were treated with either DMSO (Ctrl) or $80 \mu \mathrm{M}$ magnolol for 6 hours. Actin was used as a loading control (the actin used in Figure 3A is the same as that used in Figure 4A; the actin used in 3B is the same as those used in Figures 1A, 2B and $4 \mathrm{~B}$, as these samples were run concurrently). Each blot shown is representative of results obtained from at least three replications from three separate experiments. A and B: Representative Western blots of proteins involved in MAPK pathways. C: Graphical representation of protein expression. (*) indicates statistical significance at $P \leq 0.1$ and (**) indicates statistical significance at $P \leq 0.05$. 


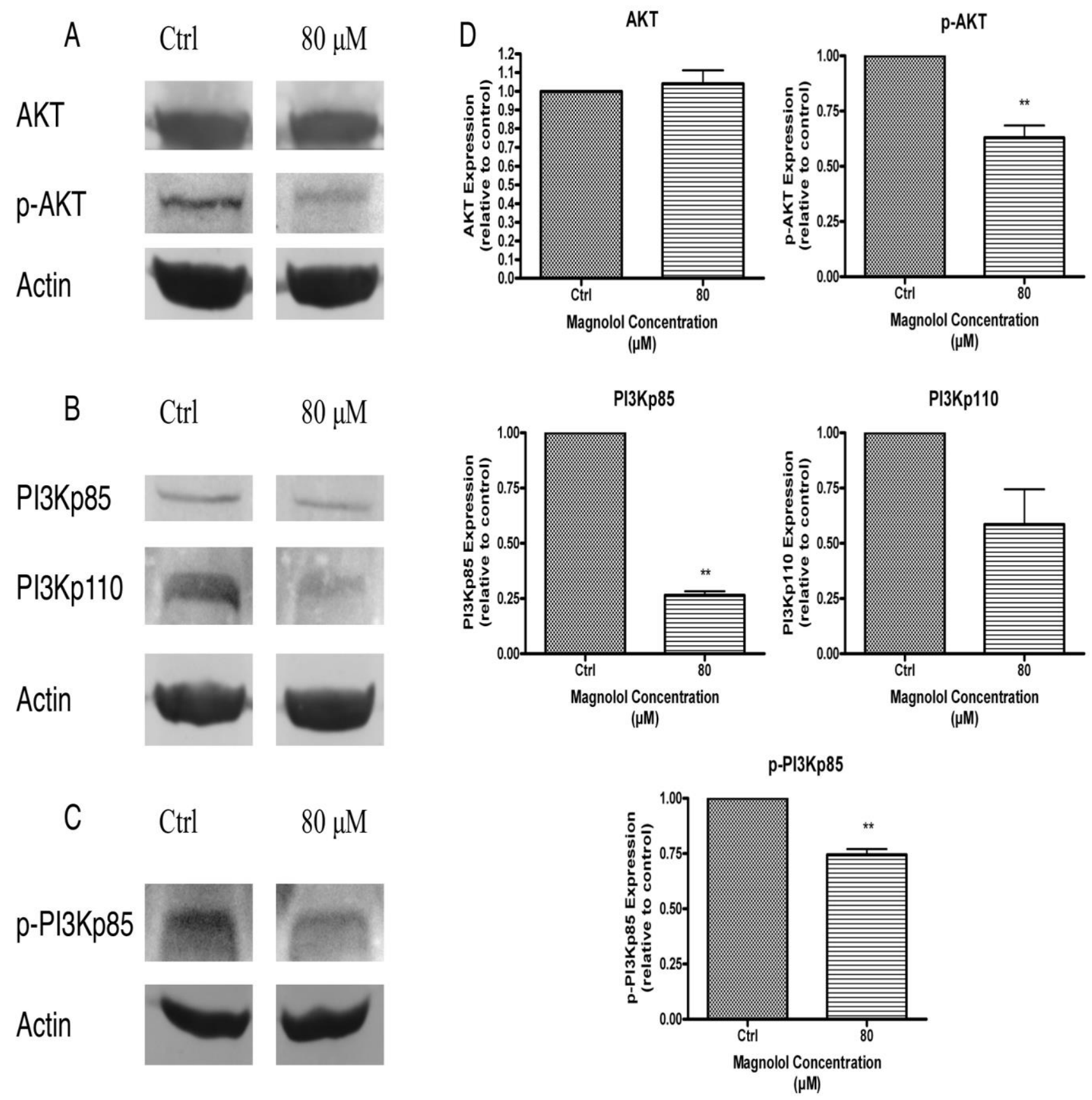

Figure 5. Magnolol affects expression of PI3K pathway proteins in PC3 cells in vitro. Cells were treated with either DMSO (Ctrl) or $80 \mu \mathrm{M}$ magnolol for 6 hours. Actin was used as a loading control (the actin used in Figure 4A is the same as that used in Figure 3A; the actin used in $4 \mathrm{~B}$ is the same as those used in Figures 1A, 2B and $3 \mathrm{~B}$, as these samples were run concurrently). Each blot shown is representative of results obtained from at least three replications from three separate experiments.

A: Representative Western blots of Akt and p-Akt proteins.

B: Representative Western blots of PI3K proteins.

C: Representative Western blot of phosphorylated PI3Kp85 protein.

D: Graphical representation of protein expression. (*) indicates statistical significance at $P \leq 0.1$ and $(* *)$ indicates statistical significance at $P \leq 0.05$. 
A

Cytosolic Nuclear

$\mathrm{D}$

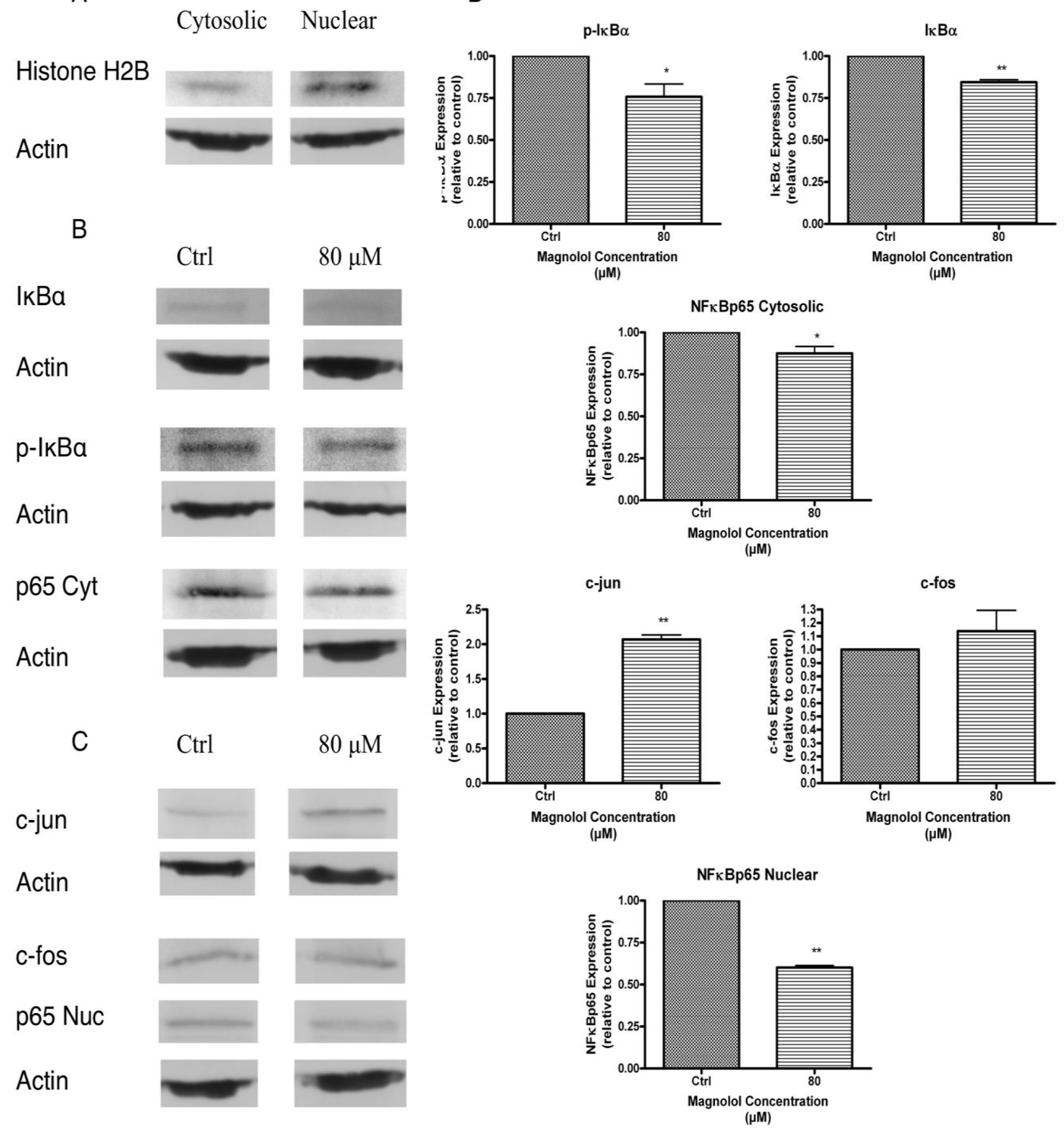

Figure 6. Magnolol affects expression of NFkB and AP-1 pathway proteins in PC3 cells in vitro. Cells were treated with either DMSO (Ctrl) or $80 \mu \mathrm{M}$ magnolol for 6 hours. Actin was used as a loading control. Each blot shown is representative of results obtained from at least three replications from three separate experiments.

A: Representative Western blot of histone H2B protein indicating nuclear protein enrichment.

B: Representative Western blots of cytosolic fraction proteins of NFKB and AP-1 pathways.

C: Representative Western blots of nuclear fraction proteins of NFKB and AP-1 pathways.

D: Graphical representation of protein expression. (*) indicates statistical significance at $P \leq 0.1$ and $(* *)$ indicates statistical significance at $P \leq 0.05$. 
Magnolol Affects Various Cellular Signaling Pathways: Following the changes observed in expression of polyamine biosynthesis and catabolism-linked proteins, several cellular signaling pathways were assayed to determine which, if any, of these pathways may be involved in the cellular response to magnolol exposure. By investigating these cellular signaling pathways and their regulators the signaling mechanism(s) through which magnolol acts can be further elucidated.

Of the MAPK pathway (Figure 4) proteins the expression of the following were significantly altered from control: p-p38 decreased to 75\%, c-Jun N-terminal kinase (JNK)-2 decreased to $81 \%$, JNK-1 decreased to $41 \%$ and p-JNK-1 increased to $116 \%$ (Figure 4). Of the PI3K pathway proteins (Figure 5) the expression of the following were significantly altered from control: PI3Kp85 decreased to $26 \%$, p-PI3Kp85 decreased to $74 \%$ and p-Akt decreased to $63 \%$ (Figure 5). The expression of the following proteins of the NFKB pathway (Figure 6) was significantly altered from control (Figure 6): NFkBp65 nuclear fraction decreased to $60 \%$, NFkBp65 cytosolic fraction decreased to $88 \%$, $\mathrm{p}$-I $\kappa \mathrm{B} \alpha$ decreased to $76 \%$ and nuclear factor of kappa-B inhibitor (IкB $\alpha$ ) decreased to $84 \%$. Finally, of the transcription factors involved in the activator protein-1 (AP-1) pathway (Figure 6) assayed, the expression of c-jun significantly increased to 207\% of the control value while no statistically significant change was observed in expression of the c-fos protein.

\section{DISCUSSION:}

In this study the effect of magnolol on the behavior of PC3 human prostate cancer cells in vitro was examined. Previously, magnolol has been found to cause alterations in expression of cell cycle proteins in DU145 and PC3 human prostate cancer cells [23]. In the present study, magnolol was found to affect cellular proliferation through alterations to proteins involved in polyamine biosynthesis and catabolism. Cellular proliferation of PC3 cells exposed to magnolol was reduced after 24 hour exposure and cell number remained virtually unchanged when cells were counted at 48 hours after exposure. Decreased protein expression of ODC in response to magnolol suggests that production of putrescine from ornithine is being limited. This is supported by reduced protein expression of AZI, and the unchanged protein expression of AZ. $\mathrm{AZ}$ acts as a non-competitive inhibitor of ODC, with AZ and ODC forming a dimer and increasing ODC degradation [26]. By increasing protein expression of $\mathrm{AZ}$ in an in vivo mouse model of upper aerodigestive tract cancers, carcinogenesis was reduced and polyamine levels decreased [27]. A reduction in AZI protein expression coupled with no significant change in AZ protein expression should affect a similar condition where available $\mathrm{AZ}$ is increased. Decreased ODC protein expression has also been associated with induction of apoptosis in MCF-7 breast cancer cells in vitro [28]. Magnolol has previously been shown by Lee et al. [11] to induce apoptosis in PC3 human prostate cancer cells. The decreased protein expression of ODC observed in the present study combined with the finding of Lee et al. [11] could indicate a similar connection between decreased ODC expression and apoptosis.

SAMDC functions in production of spermidine from putrescine and spermine from spermidine. The increase in SAMDC protein expression is opposite to that of ODC, (which decreased), suggesting that SAMDC may be compensating for the loss of ODC in driving polyamine production forward. In in vivo mouse models, an increase in SSAT protein expression 
was accompanied by increasing ODC protein expression, compensating for the increased polyamine catabolism with increased biosynthesis [29, 30]. This suggests that the decreases in SSAT and PAO in the present study may be due to a similar compensatory mechanism whereby decreased polyamine biosynthesis through reduced ODC protein expression is compensated with reduced SSAT and PAO protein expression. This results in a reduced polyamine catabolism. Inhibition of PAO has also previously been linked to the induction of apoptosis in certain leukemia cell lines in vitro [31].

In assaying the protein expression of the R1 and R2 subunits of ribonucleotide reductase, only the protein expression of the $\mathrm{R} 2$ subunit was decreased. As ribonucleotide reductase functions in converting ribonucleosides to deoxyribonucleosides, this enzyme is of key importance to cellular proliferation. R1 protein is constitutively expressed throughout the cell cycle, but R2 protein expression is most closely associated with the S-phase of the cell cycle [24, 25]. The change in the availability of the $\mathrm{R} 2$ subunit is due both to changes in protein expression as well as changes in protein degradation [24]. The R2 subunit is also recognized as the ratelimiting factor in conversion of ribonucleosides to deoxyribonucleosides, thereby acting as an important regulator of DNA synthesis with inhibition of this R2 subunit slowing down DNA synthesis [24]. As a result of its intimate ties to DNA synthesis, ribonucleotide reductase has been the target of chemotherapies in various types of cancer [24]. As magnolol has previously been shown to cause alterations in the cell cycle of PC3 human prostate cancer cells in vitro, the reduction of R2 protein expression in the current study further supports reduced proliferation and reduced ability for PC3 cells to proceed through the cell cycle in the presence of magnolol [23].

Finally, several cellular signaling pathways were assayed to determine which, if any, are affected in the cellular response to magnolol exposure. Of the MAPK pathways, magnolol has previously been shown to decrease protein expression of p38 and increase extracellular signalregulated kinase $\mathrm{p}-(\mathrm{ERK})-1 / 2$ protein expression with no significant change to protein expression of ERK-1/2 nor p-p38 in SKOV3 human ovarian cancer cells in vitro [6]. In COLO-205 human colon cancer cells in vitro magnolol exposure was associated with an increase in p-ERK-1/2 protein expression, however; protein expression of ERK-1/2 was not affected [7]. In human urinary bladder cancer 5637 cells in vitro exposed to $60 \mu \mathrm{M}$ magnolol for 3, 6 and 12 hours, an increase in protein expression of p-ERK-1/2, p-p38 and p-JNK-1/2 with no apparent change in the non-phosphorylated forms occurred [10]. In U373 human malignant glioblastoma cells in vitro exposed to magnolol, increased protein expressions of p-ERK-1/2 and p-p38 were observed after exposure to $100 \mu \mathrm{M}$ magnolol for 60 min with no change to JNK-1/2, ERK-1/2, p38 or pJNK-1/2 [32]. In VSMC human aortic smooth muscle cells in vitro no effect on ERK-1/2 or pERK-1/2 was observed in response to magnolol [9]. In the present study, the alterations to the MAPK pathways are relatively minor, with significant changes only occurring in p-p38, JNK-1/2 and p-JNK-1. The changes to MAPK protein expression is variable throughout these previous studies with no observed change to ERK-1/2 observed in any of the referenced studies and increases in protein expression of p-ERK-1/2 occurred in all studies aside from the present investigation and that of Kim et al. [9]. Inhibition of ERK-1/2 and p-ERK-1/2 has previously been associated with decreased expression of ODC in human leukemia L1210 cells in vitro, as well as the opposite, increased ERK-1/2 and p-ERK-1/2 associated with increased ODC. As no change in expression of these proteins was observed in the present study it is unlikely that they 
are involved in the decrease of ODC expression in response to magnolol observed in the present study [33]. Protein expression of p-p38 is especially variable with increases in expression observed by Chen and Lee [32] and Lee et al. [10], but no change observed by Chuang et al. [6] and a decrease in expression observed in the present study. The highly variable effect of magnolol on proteins of the MAPK pathways are likely due to these proteins being differently affected across various cell lines.

Unlike the variable nature of the effect of magnolol on the MAPK pathways, the effects of magnolol on the expression of those proteins involved in the PI3K/Akt pathway appear to be conserved across cell lines. The PI3K/Akt signaling pathway has previously been found to be involved in apoptosis-related signaling in various cancer cell lines in vitro in response to magnolol exposure $[6,11,14]$. These studies observed decreased protein expression of $\mathrm{p}$-PI3K and p-Akt with no change in protein expression of Akt. The results of the present study support those observations of the previous study involving PC3 human prostate cancer cells in that alterations to cellular viability were associated with these changes to the PI3K pathway, but show an additional change in PI3Kp85, which was not observed previously [11]. This change may be related to the higher concentration of magnolol, $80 \mu \mathrm{M}$ as opposed to $60 \mu \mathrm{M}$, used in the present study. While the PI3K pathway is typically associated with cell survival, the pathway also plays an important role in activating proliferation by controlling cyclin D and p27 activity [34]. The PI3K pathway can also control cell growth through mTOR, with decreased PI3K expression resulting in decreased cellular growth though this pathway [34]. The decreased activation of the PI3K/Akt pathway observed in the present study may affect both cellular growth and proliferation through these mechanisms.

The protein expression of NFкBp65 was decreased both in the cytoplasm and more sharply decreased within the nucleus in the present study. This suggests that there is reduced expression of this protein and that translocation of NFkBp65 into the nucleus is reduced in response to magnolol exposure. NFKB is an inhibitor of apoptosis as well as being involved in the transcription, and activation, of cyclin D [35]. Lastly, the increased protein expression of c-jun observed in the present study is contrary to the anti-proliferative effect of magnolol on PC3 human prostate cancer cells. As increased c-jun transcription is generally associated with increased cellular proliferation, it is possible that protein expression of c-jun is increased in a compensatory manner. While protein expression of c-fos was not significantly altered in response to magnolol exposure in the present study, the increased expression of c-jun may act in concert with stable expression of c-fos to activate the AP-1 pathway. While reduced expression of c-jun and c-fos is associated with decreased cellular proliferation, increased expression of these two proteins involved in the AP-1 pathway can also be an indication of apoptotic conditions, although it is unknown if there is a functional role played by the AP-1 pathway or if it is merely an associated indicator [36].

Whether such changes observed with our in vitro studies would also occur in in vivo studies remains to be determined. The bioavailability of magnolol would certainly have an influence on what would occur in an in vivo environment. In this regard, studies have shown that a $5 \mathrm{mg} / 50 \mathrm{~g}$ injection of magnolol ( 5 injections on alternate days ) in nude mice with U375 xenografts resulted in a reduction in tumor size in the mice [32]. Magnolol $(100 \mu \mathrm{M})$ was also used in in vitro assays with U375 cells [32]. This concentration is higher than that used in our study. This 
finding would suggest that magnolol can have a marked effect in an in vivo environment and would suggest that the effective concentrations to acquire this effect would be achievable .

\section{CONCLUSIONS:}

In conclusion, the present study has shown that magnolol does affect expression of proteins involved in cellular proliferation-linked activities in PC3 human prostate cancer cells. These effects were modulated through alterations in multiple cellular signaling pathways. Although the effects of magnolol on the MAPK signaling pathways are highly variable between various cancer cell types, the PI3K pathway is consistently altered and as such is a likely pathway by which magnolol affects cellular proliferation. Changes in the NFKB pathway similarly suggest that PC3 response to magnolol exposure is to reduce cellular proliferation. This study was performed using PC3 androgen-insensitive human prostate cancer cells. It would be predicted that similar observations might occur with other androgen-insensitive human prostate cancer cell lines, such as DU145 cells. However such studies with DU145 cells examining the effects of magnolol on the cellular proliferation linked activities which were evaluated in PC3 cells have not been performed. Further research is required to determine the effects of magnolol on cellular proliferation in an in vivo model, and to determine if magnolol affects cellular proliferation through alternative pathways, such as disruptions to growth factor signaling.

Abbreviations: AP-1: activator protein 1, AZ: antizyme, AZI: antizyme inhibitor, ERK: extracellular signal-regulated kinase, IкB $\alpha$ : kappa-B inhibitor, JNK: c-Jun N-terminal kinase, MAPK: mitogen-activated protein kinase, NFкB: nuclear factor of kappa-light-chain-enhancer of activated B cells, ODC: ornithine decarboxylase, PAO: polyamine oxidase, PI3K: phosphatidylinositol 3-kinase, SAMDC: S-adenosylmethionine decarboxylase, SSAT: spermidine/spermine N1-acetyltransferase

Competing Interests: The authors have no competing interests to declare.

Authors' Contributions: B. McKeown is a current graduate student in the Hurta lab and performed the research outlined in this manuscript as well as drafting the manuscript itself. R. Hurta is the primary senior investigator associated with this work and was responsible for the supervision of the research described, editing the manuscript and acquisition of grant funding required in undertaking this project and supporting the student performing the studies.

Acknowledgements: This work was supported by funding (research grant) from the Jeanne and J.-Louis Lévesque Foundation. B. McKeown is a recipient of a graduate student research assistantship from the Jeanne and J.-Louis Lévesque Foundation. R. Hurta is a recipient of the Lévesque Professorship in Nutrisciences and Health.

\section{REFERENCES:}

1. American Cancer Society. 2001. Global cancer facts and figures 2nd edition, Atlanta: American Cancer Society. 
2. Canadian Cancer Society/Canadian Cancer Society's Advisory Committee on Cancer Statistics. 2013. Canadian cancer statistics 2013. Toronto, Canada.

3. Bemis DL, Capodice JL, Costello EJ, Vorys GC, Katz AE and Buttyan R. 2006. The use of herbal and over-the-counter dietary supplements for the prevention of prostate cancer. Current Urology Reports 7:166-174.

4. Ikarashi Y, Yuzurihara M, Sakakibara I, Nakai Y, Hattori N and Maruyama Y. 2001. Effects of the extract of the bark of Magnolia obovata and its biphenolic constituents magnolol and honokiol on histamine release from peritoneal mast cells in rats. Planta Medica 67:709-713.

5. Zhang WW, Li Y, Wang XQ, Tian F, Cao H, Wang MW and Sun QS. 2005. Effects of magnolol and honokiol derived from traditional Chinese herbal remedies on gastrointestinal movement. World Journal of Gastroenterology 11:4414-4418.

6. Chuang TC, Hsu SC, Cheng YT, Shao WS, Wu K, Fang GS, Ou CC and Wang V. 2011. Magnolol down-regulates HER2 gene expression, leading to inhibition of HER2mediated metastatic potential in ovarian cancer cells. Cancer Letters 311:11-19.

7. Hsu YF, Lee TS, Lin SY, Hsu SP, Juan SH, Hsu YH, Zhong WB and Lee WS. 2007. Involvement of Ras/Raf-1/ERK actions in the magnolol-enduced upregulation of p21 and cell-cycle arrest in colon cancer cells. Molecular Carcinogenesis 46:275-283.

8. Hwang ES and Park KK. 2010. Magnolol suppresses metastasis via inhibition of invasion, migration, and matrix metalloproteinase-2/-9 activities in PC-3 human prostate carcinoma cells. Bioscience, Biotechnology and Biochemistry 74:961-967.

9. Kim HM, Bae SJ, Kim DW, Kim BK, Lee SB, Lee US, Kim CH and Moon SK. 2007. Inhibitory role of magnolol on proliferative capacity and matrix metalloproteinase- 9 expression in TNF- $\alpha$-induced vascular smooth muscle cells. International Immunopharmacology 7:1083-1091.

10. Lee SJ, Cho YH, Park K, Kim EJ, Jung KH, Park SS and Moon SK. 2008. Magnolol elicits activation of the extracellular signal-regulated kinase pathway by inducing p27KIP1-mediated G2/M-phase cell cycle arrest in human urinary bladder cancer 5637 cells. Biochemical Pharmacology 75:2289-2300.

11. Lee DH, Szczepanski MJ and Lee YJ. 2009. Magnolol induces apoptosis via inhibiting the EGFR/PI3K/Akt signaling pathway in human prostate cancer cells. Journal of Cellular Biochemistry 106:1113-1122.

12. Lin SY, Chang YT, Liu JD, Yu CH, Ho YS, Lee YH and Lee WS. 2001. Molecular mechanisms of apoptosis induced by magnolol in colon and liver cancer cells. Molecular Carcinogenesis 32:73-83.

13. Lin SY, Liu JD, Chang HC, Yeh SD, Lin CH and Lee WS. 2002. Magnolol suppresses proliferation of cultured human colon and liver cancer cells by inhibiting DNA synthesis and activating apoptosis. Journal of Cellular Biochemistry 84:532-544.

14. Rasul A, Yu B, Khan M, Zhang K, Igbal F, Ma T and Yang H. 2012. Magnolol, a natural compound, induces apoptosis of SGC-7901 human gastric adenocarcinoma cells via the mitochondrial and PI3K/Akt signaling pathways. International Journal of Oncology 40:1153-1161. 
15. Ho JHC and Hong CY. 2012. Cardiovascular protection of magnolol: cell-type specific and dose-related effects. Journal of Biomedical Science 19:70.

16. Lin SP, Tsai SY, Chao PDL, Chen YC and Hou YC. 2011. Pharmacokinetics, bioavailability, and tissue distribution of magnolol following single and repeated dosing of magnolol to rats. Planta Medica 77:1800-1805.

17. Tsai TH, Chou CJ and Chen CF. 1996. Pharmacokinetics and brain distribution of magnolol in the rat after intravenous bolus injection. The Journal of Pharmacy and Pharmacology 48:57-59.

18. Li N, Song Y, Zhang W, Wang W, Chen J, Wong AW and Roberts A. 2007. Evaluation of the in vitro and in vivo genotoxicity of magnolia bark extract. Regulatory Toxicology and Pharmacology 49:154-159.

19. Liu Z, Zhang X, Cui W, Zhang X, Li N, Chen J, Wong AW and Roberts A. 2007. Evaluation of short-term and subchronic toxicity of magnolia bark extract in rats. Regulatory Toxicology and Pharmacology 49:160-171.

20. Perez-Leal O and Merali S. 2012. Regulation of polyamine metabolism by translational control. Amino Acids 42:611-617.

21. Igarashi K and Kashiwagi K. 2009. Modulation of cellular function by polyamines. The International Journal of Biochemistry \& Cell Biology 42:39-51.

22. Nowtoarski SL, Woster PM and Casero RA Jr. 2013. Polyamines and cancer: implications for chemoprevention and chemotherapy. Expert Reviews in Molecular Medicine 15:e3

23. McKeown B, McDougall L, Catalli A and Hurta R. 2014. Magnolol causes alterations in the cell cycle in androgen insensitive human prostate cancer cells in vitro by affecting expression of key cell cycle regulatory proteins. Nutrition and Cancer 66:1154-1164.

24. Guarino E, Salguero I and Kearsey SE. 2014. Cellular regulation of ribonucleotide reductatase in eukaryotes. Seminars in Cell \& Developmental Biology 30:97-103.

25. Norlund, P and Reichard P. 2006. Ribonucleotide reductases. Annual Review of Biochemistry 75:681-706.

26. Pegg AE. 2006. Regulation of ornithine decarboxylase. Journal of Biological Chemistry 281:14529-14532.

27. Feith DJ, Pegg AE and Fong LYY. 2013. Targeted expression of ornithine decarboxylase antizyme prevents upper aerodigestive tract carcinogenesis in p53-deficient mice. Carcinogenesis 34:570-576.

28. Arisan ED, Obakan P, Coker A and Palavan-Unsal N. 2012. Inhibition of ornithine decarboxylase alters the roscovitine-induced mitochondrial-mediated apoptosis in MCF-7 breast cancer cells. Molecular Medicine Reports 5:1323-1329.

29. Kee K, Foster BA, Merali S, Kramer DL, Hensen ML, Diegelman P, Kisiel N, Vujcic S, Mazurchuk RV and Porter CW. 2004. Activated polyamine catabolism depletes acetylCoA pools and suppresses prostate tumor growth in TRAMP mice. Journal of Biological Chemistry 279:40076-40083.

30. Tucker JM, Murphy JT, Kisiel N, Diegelman P, Barbour KW, Davis C, Medda M, Alhonen L, Jänne J, Kramer DL, Porter CW and Berger FG. 2005. Potent modulation of 
intestinal tumorigenesis in Apcmin/+ mice by the polyamine catabolic enzyme spermidine/spermine N1-acetyltransferase. Cancer Research 65:5390-5398.

31. Wallace HM and Fraser AV. 2004. Inhibitors of polyamine metabolism: review article. Amino Acids 26:353-365.

32. Chen LC and Lee WS. 2013. p27/kip1 is responsible for magnolol-induced U373 apoptosis in vitro and in vivo. Journal of Agricultural and Food Chemistry 61:2811-2819.

33. Flamigni F, Facchini A, Capanni C, Stefanelli C, Tantini B and Caldarera CM. 1999. p44/42 mitogen-activated protein kinase is involved in the expression of ornithine decarboxylase in leukaemia L1210 cells. Biochemical Journal 341:363-369.

34. Vivanco I and Sawyers CL. 2002. The phosphatidylinositol 3-kinase-Akt pathway in human cancer. Nature Reviews Cancer 2:489-501.

35. Karin M, Cao Y, Greten FR and Li ZW. 2002. NF-kB in cancer: from innocent bystander to major culprit. Nature Reviews Cancer 2:301-310.

36. Shaulian E and Karin M. 2002. AP-1 as a regulator of cell life and death. Nature Cell Biology 4:E131-E136. 